\title{
Presence Disparity in Mixed Presence Collaboration
}

Anastasia Bezerianos

NICTA and HxI Initiative

Australian Technology Park

Bay 15 Locomotive Workshop

Eveleigh, NSW 1430

Australia

a.bezerianos@nicta.com.au

\section{Gregor McEwan}

NICTA and HxI Initiative

Australian Technology Park

Bay 15 Locomotive Workshop

Eveleigh, NSW 1430

Australia

gregor.mcewan@nicta.com.au

\begin{abstract}
We present the design of an experiment investigating presence disparity in mixed presence collaboration using digital tabletops. In an attempt to verify previous work and relate their results, we examined different presence representations of remote collaborators: audio, video, telepointers and video arms. Our early results show some interesting trends that we are currently investigating in more detail through further analysis of our data.
\end{abstract}

\section{Keywords}

Tabletop, mixed presence, collaboration, presence disparity

\section{ACM Classification Keywords}

H.5.3 [Group and Organisation Interfaces]:

Computer supported cooperative work

\section{Introduction}

Mixed presence collaboration combines distributed and collocated collaboration, i.e. there are multiple distributed sites, each with a collocated group. While purely collocated collaboration and purely distributed collaboration are each the subject of rich bodies of research, the combination of collocated and distributed is less well explored. 
Mixed presence collaboration faces many of the challenges of both collocated collaboration (e.g. orientation issues [6]), and distributed collaboration (e.g. workspace awareness [5]), but also faces unique challenges arising from the mixture of the two. Tang et al. [9] first coined the term presence disparity to refer to collaborators' tendency to interact more with collocated people in preference to remote team members.

Tang et al.'s initial informal observations of presence disparity [9] were also supported in their later experimentation [10]. One condition in this experiment incorporated video arms - as participants reach over their display, the remote site is shown the video of their arms and hands. In the experimental trials with video arms, distributed interactions were more balanced with the collocated interactions, though there was still a distinct preference for collocated interaction. Presence disparity was mitigated by increasing the presence of the remote participants.

figure 1: Physical experiment setup in one of the rooms. Video arms of remote participants can be seen on the screen (green). The screen showing remote video is partially visible in the background.
A more recent study by Epps et al. [4] reported seemingly contradictory results. Their results showed more interaction with remote group members than collocated members. To try and explain the seeming contradiction, we looked to the differences between the two experiments. They used a count of remote and collocated communication events while Tang et al. [10] based their report on qualitative observations.

Moreover, Epps et al.'s setup involved a video link, while Tang et al. had two conditions: audio only, and audio with video arms. Finally, the number of participants and the tasks performed were different. The differences make it hard to compare the results.
To investigate further this idea of the relationship between remote presence mechanisms and presence disparity, we conducted a controlled laboratory experiment to try and unify the above results. The experiment involved collaborative tasks in a mixed presence setting with varied remote presence mechanisms (audio, video, telepointers, and video arms) to see their impact on presence disparity. This report describes our very early results.

\section{Experimental Design}

The purpose of the experiment was to investigate how remote presence mechanisms affected mixed presence collaboration. Our two experimental hypotheses were:

1. Interaction within a mixed presence group changes depending on the remote presence information mechanisms provided.

2. The imbalance between remote and collocated interactions, or presence disparity, is less when there is more remote presence information provided.

To test the hypotheses we set up a mixed presence scenario with two sites and two people at each site. We did two trials with each of four remote presence conditions (eight trials in total).

Physical Setup (figure 1)

We configured two rooms, each with a horizontal digital tabletop display and audio-video conferencing facilities. The digital tabletops were $101.9 \times 57.4 \mathrm{~cm}$ (displayable area) LCD screens with a 16:9 aspect ratio. They both had an overlay providing dual touch input and were 
positioned horizontally approximately $1 \mathrm{~m}$ above the ground, for comfortable interaction while standing.

The audio-video conferencing equipment consisted of a 19" LCD monitor, a standard PC webcam, an echo cancelling microphone, and set of standard computer speakers for communication between sites. These were all placed at one of the shorter sides of the digital tabletop, with a gap of approximately $1 \mathrm{~m}$ so that participants could walk around the tabletop. An overhead webcam at each site pointed down at the

table captured images for the video arms. These

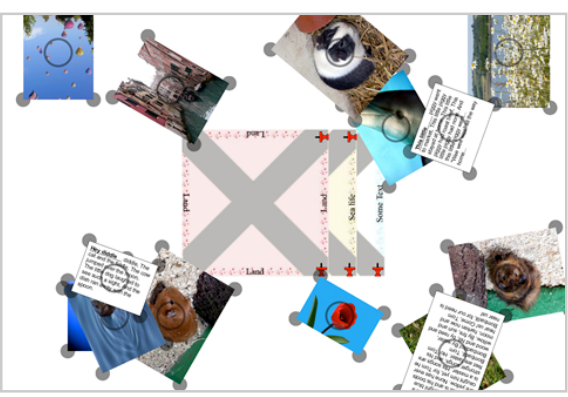

figure 2: Screenshot of the training task showing pictures (e.g. top left), text (e.g. bottom right) and layout containers (center of screen with grey crosses). as were positioned above head height so as to

not interfere with the participants' movements.

\section{Software Setup}

The software used displayed a variable number of objects - pictures, text or layouts - on the tabletop (figure 2). The display was replicated at each site so all four participants saw the same view. Picture and text objects could be translated and rotated freely using the algorithm outlined by Kruger et al. [7], and resized using widgets attached to the objects. Layout objects served as containers for other objects. Participants interacted with a set of these objects to perform layout tasks.

Communication and Presence Conditions

We had four presence conditions, listed here in order of increasing remote presence information:

1. Audio: There was only an audio channel available for communication between sites. Telepointers, showing the locations of remote participants' touches, were displayed on the table.
2. Audio and Video: In addition to the audio communication and telepointers, a full screen video of the remote site was shown on the monitor.

3. Audio and Video Arms: As participants reached over the tabletop, the shapes of their arms were drawn on the remote tabletop, as seen in . Audio and telepointers were still in effect. There was no video.

4. Audio, Video and Video Arms: As in condition 3 but with a video display of remote participants.

Participants

We ran a total of 32 participants in eight groups of four ( 2 groups per condition). All participants were full time professionals in a variety of roles - such as research, administration, legal, human resources, and finance within a technology research company. In all groups participants had previously worked closely with at least one other member of their group, and in most cases with all other members of the group (7/8 groups). Group familiarity and computer experience was captured in a pre-trial questionnaire. Participants (12 female) were aged between 24 and 50 (average 35 years old). They all received a coffee voucher and snacks in return for their participation.

Experimental Procedure and Tasks

After completing a brief demographic questionnaire, participants were introduced to the digital tabletops and software. They were then split into two groups to perform a training layout task (figure 2) with the presence condition setup for their trial.

Each group completed two tasks: a text and a picture layout task. The order of task presentation was varied. 
After completion of the first task, one participant from each site swapped location, so that pairs were mixed.

- Text task. This task was similar to one used by Birnholtz et al. [2]. Participants were shown sixteen text articles and one layout object. Each text article was a shortened news article with the nouns replaced with words from four categories - fruit, vegetables, carbohydrates and protein. Each participant was assigned a category and given a list of four words from that category, unknown to the rest of the group. The group was given the task of creating a single layout of the text articles (not all articles could fit on the layout), while each individual had a private goal of getting as many of his or her words in that final layout. To enhance communication all participants had to agree on the final layout, while the person with the most words in the final layout was given a movie voucher.

- Picture task. This task was modelled loosely on one designed by Scott et al [8]. Participants were given 50 pictures from a popular movie, and three layouts with different themes. Participants were required to select five representative images for each theme and use them to create a pleasing layout.

After completing both tasks, participants took part in a semi-structured group interview, and filled out a second questionnaire eliciting their opinions on the task at hand and their views on the quality of communication and coordination with remote and collocated partners.

Measures

We measured a broad range of data for later analysis:
- Pre-trial questionnaire. The questions measured demographic data, such as age, familiarity with various technologies (e.g. videoconferencing and touch input) as well as group familiarity.

- Video recording. We recorded the trials and the post-trial nterviews. We are currently analysing the trial videos using semi-structured coding methods.

- Logging. Automated logging collected interactions with the tabletop software. The log files were used to generate videos of the tabletop for later analysis.

\section{- Post-trial semi-structured group interviews.}

The interviews were conducted with the group of four together. In the interview we prompted the participants to talk about their experience of aspects of collaboration, communication and interaction with the workspace. We asked them to compare the experience of interacting with remote collaborators with that of collocated collaborators.

- Post-trial questionnaires. The questionnaires were a chance to elicit individual and structured responses. The majority of questions were Likert scale responses, based on existing questionnaires [5][8][10] investigating workspace awareness, collaboration, and overall workspace interaction experience. We modified the questions to address remote and collocated separately when applicable, enabling users to directly compare their remote and collocated experiences. The questionnaire, and its use in this and a later experiment, is described in Bezerianos et al. [1].

\section{Early Results}

So far, we have examined our logged data and analysed the post-trial questionnaires. Here we report on some of the interesting early results and trends. 


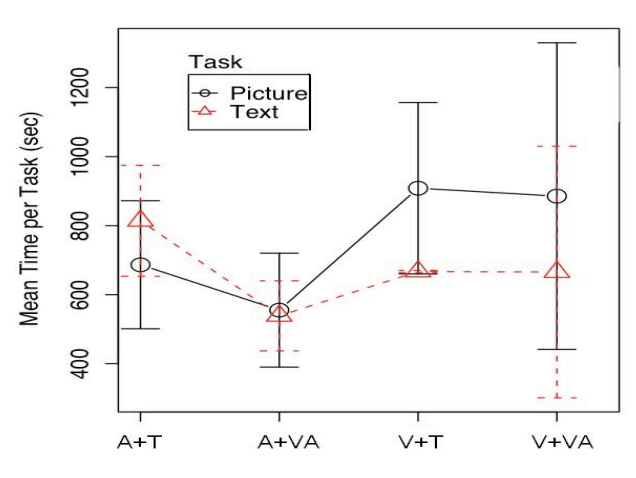

figure 3: Mean completion time (sec) for the four presence conditions.

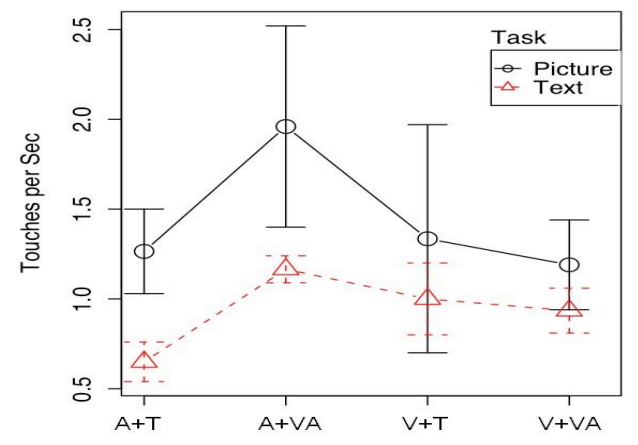

figure 4: Touch rate per second for each of the four presence conditions.
Analysis of variance showed a significant difference (all $\mathrm{p}<0.01$ ) between how people perceived awareness, coordination and communication with local and remote participants, for all questions that compared the two. This supports the findings on presence disparity [10].

However, the questionnaires show no significant effect of ranking based on presence condition, though there is a trend for participants in higher presence conditions (video and video arms) to rank their remote partners higher, especially in questions related to awareness of actions on the workspace [1]. We also normalised the Likert scale responses to remove individual rating biases, by subtracting the response for collocated collaborators from that for remote collaborators. Although no statistical difference was found between conditions, we observed that participants in high presence conditions tended to also rank their collocated partners higher than in lower presence conditions, thus balancing out distances between conditions, indicating the possible existence of a confound effect.

The lack of significance is disappointing, but could be explained by the fact that our groups had high familiarity (work and social contact as a group close to once a week on average) and so had an established relationship and understanding that may have balanced out poor remote presence. We are currently analysing the video recordings to determine how groups changed their strategies and behaviour to compensate for poorer remote presence information.

\section{It is interesting to note that participants in high} presence conditions commented on it positively during the interview: "I did not often look at it [remote video], but there was a sense of presence", "I could use the shadow of my arm to point", "I knew it was them that did something when I saw the green hand".

To gain a better picture of collaborative tendencies, we also looked at task completion time, as well as the interaction touches per second for all presence conditions. Figure 3 shows the completion times organised by presence condition. There was no significant difference between times. We believe that the completion times were roughly the same for two reasons. Firstly, we specified no time limits and so there was no pressure to finish, and secondly some groups greatly enjoyed the tasks and spent a long time arranging the content in interesting ways.

Interestingly (figure 4) we found that the condition with least touches was that of least presence (audio + telepointers) followed by the highest presence condition (with video and video arms). Our working hypothesis is that groups performed tasks more economically (less touches) with highest presence of remote participants as they had a better understanding of actions of other participants. The exception is the very low presence condition, where participants had to extensively coordinate verbally to make-up for poor remote presence. We are currently analysing the videos of participants to verify this hypothesis and discover clues as to the cause of this exception.

\section{Current and Future work}

We have conducted an exploratory study investigating presence disparity in mixed presence groups with high between-group familiarity. Early results show that our participants perceived their remote collaborators similarly across presence conditions, even if they appreciated the enhanced presence mechanisms. 
Nevertheless, there are indications that workspace interaction was affected by presence condition.

We are currently in the process of analysing video from our experiment, to better compare our results to the studies of Epps et al. [4] and Tang et al. [10]. We are looking at both quantitative metrics (such as communication frequency [4]), as well as qualitative observations on communication patterns [10].

Furthermore, in our video analysis we are examining other parameters that might be affected by the different presence representations, such as participant movement around the table, use of space and territory formation already investigated in collocated settings [8], subgroup formation, and participant strategies for completing the task.

We hope that our continued analysis of the data will reveal more insights into how presence technology affects mixed presence collaboration. We also hope to gain more general understanding of how groups interact in mixed presence collaboration settings.

\section{Acknowledgements}

We thank our colleagues at CSIRO, DSTO and NICTA for their feedback and assistance with designing, running and analysing this study. We also thank Stephanie Smale for reviewing this paper.

This research was conducted within the HxI Initiative an Australian research initiative led by the Commonwealth Scientific Research Organisation (CSIRO), Defence Science and Technology Organisation (DSTO) and National ICT Australia (NICTA).

\section{References}

[1] Bezerianos, A, Wessels A, and McEwan, G.

Collaboration experience in mixed presence negotiation tasks. Submitted to $C H I^{\prime} 08$, WIP.

[2] Birnholtz, J., Grossman, T., Mark, C., and Balakrishnan, R. 2007. An exploratory study of input configuration and group process in a negotiation task using a large display. In CHI 2007, 91-100.

[3] Hauber, J., Regenbrecht, H., Billinghurst, M., and Cockburn, A. 2006. Spatiality in videoconferencing: trade-offs between efficiency and social presence. In CSCW 2006, 413-422.

[4] Epps, J., Close, B. 2007. A study of co-worker awareness in remote collaboration over a shared application. CHI Extended Abstracts 2007, 2363-2368.

[5] Gutwin, C., Greenberg, S., and Roseman, M. 1996. Workspace Awareness in Real-Time Distributed Groupware: Framework, Widgets, and Evaluation. In HCI on People and Computers XI, 281-298.

[6] Kruger, R., Carpendale, S., Scott, S. D., and Greenberg, S. 2003. How people use orientation on tables: comprehension, coordination and communication. In GROUP '03, 369-378.

[7] Kruger, R., Carpendale, S., Scott, S. D., and Tang, A. 2005. Fluid integration of rotation and translation. In CHI 2005, 601-610.

[8] Scott, S. D., Carpendale, S., and Habelski, S. 2005. Storage Bins: Mobile Storage for Collaborative Tabletop Displays. In IEEE CG \& Applications, 25(4), 58-65.

[9] Tang, A., Boyle, M., and Greenberg, S. 2005. Display and presence disparity in Mixed Presence Groupware. In Journal of Research and Practice of Information Technology, Vol. 37, No. 2, 71-88.

[10] Tang, A., Neustaedter, C. \& Greenberg, S. 2006. VideoArms: embodiments in mixed presence groupware. In BCS-HCI British HCI. 